\title{
Elaboración de una Escala de Apoyo Social (EAS) para adultos*
}

\section{Elaboration of a Social Support Scale for adults}

Recibido: diciembre 5 de 2012 | Revisado: enero 20 de 2013 | Aceptado: enero 27 de 2013

\author{
JoAQuina PALOMAR LeVer ** \\ Graciela LORENA MATUS García *** \\ AMPARO VICTORIO ESTRADA \\ Universidad Iberoamericana, Ciudad de México
}

\section{RESUMEN}

El apoyo social se ha definido como las transacciones interpersonales instrumentales que implican ayuda en todas sus vertientes, tales como emocional, económica, implícita, explícita, etc. La presente investigación tuvo como objetivo elaborar una escala que midiera el apoyo social para la población mexicana en situación de pobreza. Se tomaron como base dos instrumentos: la Multidimensional Scale of Perceived Social Support y el Inventory of Socially Supportive Behaviors. La muestra la conformaron 913 personas de ambos sexos. Un análisis factorial permitió observar la emergencia de tres componentes: 1) apoyo social, 2) apoyo familiar y 3) apoyo de amigos. Los tres factores explican el $66.24 \%$ de la varianza y tienen índices de confiabilidad adecuados que oscilan entre 0.71 y 0.94 .

Palabras clave autores

Apoyo social, apoyo familiar, apoyo de amigos, escala, pobreza.

Palabras clave descriptores

Psicología de la Salud, Psicometría, Validez, Confiabilidad, Análisis Factorial.

Para citar este artículo: Palomar, J., Matus, G. L. \& Victorio, A. (2013). Elaboración de una Escala de Apoyo Social (EAS) para adultos. Universitas Psychologica, 12(1), 129-137.

Investigación realizada con al apoyo de Sedesol, CONACYT y Universidad Iberoamericana.

** Profesor-investigador de tiempo completo. Departamento de Psicología.E-mail: joaquina.palomar@ uia.mx

*** Profesores del Departamento de Psicología. E-mails: lorenamatus@hotmail.com, avictorioe@gmail. com

\section{A B S T R A C T}

The social support has been defined as the interpersonal instrumental transactions that involve help in any of its forms, such like emotional, economic, implicit, explicit, etc. The present research was aimed to devise a scale to measure the perception of social support in Mexicans living in poverty. The scale was based on two instruments: 1) The Multidimensional Scale of Perceived Social Support, and 2) The Inventory of Socially Supportive Behaviors. The sample was conformed by 913 people, men and women. A factorial analysis resulted in an internal structure of three components: 1) Social support, 2) Family support, and 3) Support from friends. The three factors explain $66.24 \%$ of the total variance and have adequate alpha coefficients that oscillate between 0.71 and 0.94 .

Key words authors:

Social support, family support, support from friends, scale, poverty.

Key words plus:

Health Psychology, Psychometry, Validity, Reliability, Factorial Analysis. 


\section{Introducción}

El apoyo social ha sido definido como las transacciones interpersonales que implican ayuda en todas sus vertientes: moral, económica, no verbal, verbal, implícita, explícita, emocional, etc. (Armstrong, Birnie-Lefcovitch \& Ungar, 2005; Guzmán, Huenchuan \& Montes de Oca, 2003). En este sentido, los especialistas han categorizado de distintas formas este constructo; por ejemplo, Guzmán et al. (2003) distinguen entre fuentes de apoyo informales y formales. Para Armstrong et al. (2005), el apoyo social puede ser instrumental, emocional, de información, de ayuda tangible, de interacciones sociales positivas, de afecto y de estima.

Kahn y Antonucci definieron al apoyo social en 1980 como las transacciones interpersonales que implican ayuda, afecto y afirmación. Este conjunto de transacciones interpersonales, al que también se denomina con el término genérico de "transferencias", se presentan como un flujo de intercambio y circulación de recursos, acciones e información de tipo material, instrumental, emocional y cognitivo. Dentro de los materiales se encuentran el dinero, la alimentación y el alojamiento. Los instrumentales están relacionados con el cuidado; los apoyos emocionales tienen que ver con los afectos, la escucha, la compañía y el reconocimiento; y, finalmente, los cognitivos hacen referencia al intercambio de experiencias y consejos (Guzmán et al., 2003). Estos apoyos son percibidos cualitativamente de forma diferente de acuerdo al contexto; por ejemplo, muchos teóricos han enfatizado que los estresores, las características sociales, la personalidad, los atributos del receptor del apoyo social, la red social, los tipos y fuentes del apoyo social, la calidad de la relación y la reciprocidad entre los miembros de una comunidad, influyen en la forma como funciona el apoyo social (Adler de Lomnitz, 1993; Ahluwalia, Dodds \& Baligh, 1998; Antonucci \& Jackson, 1990; Daniels \& Guppy, 1997; Green \& Rodgers, 2001). Igualmente, Kim (2000 citado en Guzmán et al., 2003) se plantea que cuando el intercambio de apoyos es recíproco se generan efectos psicológicos positivos en las personas participantes, lo cual no sucede si el intercambio no es balanceado.
En las sociedades en las que se da relevancia a los logros comunitarios, el intercambio y la recepción de apoyo suelen percibirse de manera más positiva que en las culturas individualistas, donde los valores que se promueven (tales como la independencia, la autoconfianza y el control interno) entran en contradicción con la búsqueda de apoyo (Barrón, 1996; Cutrona, Hessling \& Suhr, 1997; Green \& Rodgers, 2001; Thomson, Lutz \& Lakey, 1999).

Las personas que son optimistas, asertivas, que poseen una alta autoestima, habilidades sociales adecuadas y que son extrovertidas, tendrán más probabilidad de percibir altos niveles de apoyo de diversos grupos sociales y lograrán sentirse más satisfechas con ello (Palomar \& Cienfuegos, 2007). Del mismo modo, cuando sus expectativas respecto al apoyo no se cumplen, frecuentemente lo explican como situaciones pasajeras. Por el contrario, las personas con una autoestima baja, poco optimistas, con un locus de control externo, aquellas que tienden a la depresión y, en general, las que se sienten menos apoyadas socialmente, se perciben de manera negativa respecto a sus habilidades sociales, dando como resultado pobres e inseguras relaciones con limitado acceso al apoyo social (Palomar \& Cienfuegos, 2007).

Asimismo, fuertes niveles de ansiedad y estrés menoscaban las capacidades sociales de los individuos, debido a que se perciben ineficientes para mantener relaciones sólidas y seguras, con el consecuente deterioro de su bienestar y el limitado acceso al apoyo social. Esto origina a su vez, que se acentúen los niveles de estrés, provocando un círculo vicioso que puede perjudicar de forma significativa a los sujetos con recursos económicos escasos, que son precisamente los que están más expuestos y son más vulnerables a estas situaciones (Acuña \& Bruner, 1999; Aduna, 1998; Daniels \& Guppy, 1997; Green \& Rodgers, 2001; Gurung, Sarason \& Sarason, 1997; Sarason, Levine, Bashman \& Sarason, 1983; Sarason, Pierece \& Sarason, 1990; Singh \& Pandey, 1990; Van Baarsen, 2002).

En trabajos realizados con poblaciones pobres de México, Montes de Oca (2000) encontró que cuando los apoyos institucionales disminuyen o desaparecen, la red de apoyo informal (familia, 
amigos y otros) tiende a activarse. Aunado a esto, estos grupos informales pueden verse seriamente dañados cuando ocurren crisis graves. Los actores que intervienen en dichas crisis sufren mermas extremas de sus propios recursos, dejando a los grupos en una situación altamente precaria.

En el estudio realizado por Palomar y Cienfuegos (2007), se distinguieron las principales fuentes de apoyo percibidas en tres grupos socioeconómicos diferentes -pobres extremos, pobres moderados y no pobres- en México. Encontraron que la principal fuente de apoyo para la mayoría de los sujetos son los padres y hermanos, seguidos por la Iglesia y la religión. La tercera fuente de apoyo para esta muestra fue la familia extendida que comprendía a los abuelos y tíos. Subsiguientemente, se encontró el apoyo proporcionado por los amigos y vecinos.

$\mathrm{Al}$ evaluar las diferencias específicas entre los sujetos de los tres niveles socioeconómicos en el estudio, Palomar y Cienfuegos (2007) notaron que los pobres extremos perciben poco apoyo de la familia y de los amigos; pero al igual que los pobres moderados, perciben altos niveles de apoyo por parte de la gente de la comunidad en la que viven. Estos resultados son consistentes con lo postulado por Abello, Madariaga y Hoyos (1997) respecto a que la pobreza es un problema que suele afectar a diversos miembros de la misma familia, de tal forma que la escasez de recursos puede impedir que la ayuda entre estos se lleve a cabo, particularmente la referida al apoyo económico. Ante este hecho, se suele buscar ayuda de otros grupos sociales, como los vecinos, con quienes presumiblemente no se tienen lazos tan estrechos pero que, debido a la cercanía física y a la posibilidad de interacción y reciprocidad, se convierten en una fuente de apoyo sumamente importante para los que menos tienen (Ahluwalia et al., 1998).

Es importante destacar que, en la investigación citada, todos los grupos del estudio (pobres extremos, pobres moderados y no pobres) subrayan la importancia relativa de la familia nuclear como fuente de apoyo. Por tanto, si bien los pobres extremos, en comparación con los no pobres y los pobres moderados, consideran percibir poco apoyo de ella; si se compara con el apoyo percibido de la Iglesia o la familia extendida, el apoyo brindado por la familia nuclear es mayor (Palomar \& Cienfuegos, 2007). Esta conclusión coincide con la de Sousa (2005) sobre el apoyo social percibido que brinda la familia. Igualmente, en numerosas investigaciones se ha reportado que el apoyo social es un recurso que permite afrontar adecuadamente las situaciones adversas (Aduna, 1998; Daniels \& Guppy, 1997; Green \& Rodgers, 2001; Sarason et al., 1983).

La presente investigación tuvo como meta construir una escala que midiera el apoyo social. Para tal efecto, se revisaron numerosos trabajos publicados, tanto en lengua inglesa como en idioma español, tales como el de Bernal, Maldonado y Scharron (2003), sin embargo, la mayoría de ellos no evaluaban todas las dimensiones del apoyo social que se querían medir; de ahí que se decidió, tomando como base la literatura y las escalas ya existentes, construir una nueva escala. Así, la nueva escala que emana de este trabajo pone énfasis en las características culturales, evaluando además, al apoyo social desde una perspectiva amplia cuyas dimensiones no son estimadas en las escalas existentes desarrolladas en México.

\section{Método}

\section{Participantes}

El objetivo de la primera etapa fue examinar el comportamiento de los ítems en una muestra piloto, mientras que en la segunda etapa el objetivo fue la aplicación en la muestra final. Estas etapas se describen a continuación.

\section{Etapa 1 del estudio}

Se trabajó con una muestra de ambos sexos de adultos, pertenecientes a hogares de nivel socioeconómico bajo y medio bajo, que habitan en zonas urbanas de la ciudad de México. Participaron 189 sujetos, de los cuales 107 fueron mujeres y 82 hombres, con una media de edad de 32.67 años. El tamaño de muestra fue considerado tomando 
como base que hubiera al menos cinco participantes por reactivo aplicado, para que los análisis psicométricos cumplieran con el supuesto básico (Nunnally, 2005).

\section{Etapa 2 del estudio}

Los participantes fueron pobres extremos, beneficiarios del programa de desarrollo humano "Oportunidades" y los cónyuges que estuvieron presentes. Los datos de la presente investigación forman parte de un estudio más amplio en donde la pobreza se define como un síndrome situacional en el que se asocian el infraconsumo, la desnutrición, las condiciones precarias de vivienda, los bajos niveles educacionales, las malas condiciones sanitarias, una inserción inestable en el aparato productivo, un cuadro actitudinal de desaliento con poca participación en los mecanismos de integración social y quizá, la adscripción a una escala particular de valores diferenciada en alguna medida de la del resto de la sociedad (Madariaga \& Sierra, 2000).

En esta etapa del estudio tomaron parte 602 hogares que se incorporaron al Programa Oportunidades desde el año 2002 hasta la fecha del levantamiento. La muestra fue seleccionada de un universo de 5.512 hogares. Para la inclusión a la muestra, se tuvieron dos criterios: que estuvieran activos en el programa y que tuvieran dos mediciones del nivel socioeconómico, una al ingresar al programa y la segunda realizada tres años después. Este último fue necesario debido a que, como ya se mencionó líneas arriba, los datos de la presente investigación forman parte de un estudio más amplio.

Los participantes vivían en las áreas urbanas de los estados de Guanajuato, Estado de México,

1 El programa de Desarrollo Humano Oportunidades es una iniciativa del ejecutivo federal que tiene como objetivo contribuir a que las familias que viven en extrema pobreza salgan de esta condición. Dirige sus acciones a impulsar y fortalecer el desarrolo de las capacidades individuales y colectivas de la población beneficiada. Mediante transferencias de ingreso e inversión en salud, nutrición y educación, el programa busca romper el círculo intergeneracional de la pobreza en México. Tiene alrededor de 5 millones de hogares beneficiarios, siendo en todos ellos las mujeres las que son titulares del programa y reciben el apoyo económico (salvo raras excepciones como en el caso de incapacidad).
Morelos, Tlaxcala, Guerrero, Michoacán, Veracruz y Puebla. De los 1.257 hogares que cubrieron estos criterios, fueron seleccionados aleatoriamente 600 , más 100 como muestra de reemplazo. Se encuestaron a 602 titulares del programa y a 311 esposos; de estos solo fue posible realizar el estudio con aproximadamente el $50 \%$ debido a que un buen porcentaje de ellos trabaja o había migrado a otro estado o a Estados Unidos. Asî mismo, algunas titulares eran viudas, separadas o madres solteras. Estos cónyuges fueron incorporados debido a que se buscó estudiar el fenómeno en la pareja que constituye el hogar y no solamente con las mujeres beneficiarias.

De la muestra examinada el $34.8 \%$ corresponde a los esposos de las titulares, mientras que el $65.2 \%$, a las mujeres. Respecto a las edades, el promedio fue de 43.71 años con una desviación estándar de 12.58 años.

Respecto al ingreso, las personas dijeron obtener mensualmente \$1.285 MXN (106 USD) en promedio por persona y que su ingreso familiar mensual asciende a $\$ 2.284$ MXN (188 USD). Con esta cantidad de dinero viven en promedio 4.62 personas en la familia y lo invierten en alimento (41.1\%), gastos escolares $(28.1 \%)$ y ropa y calzado $(19.4 \%)$.

De acuerdo con el área laboral, la mayoría de las mujeres de la muestra se dedican al hogar, es decir son amas de casa, y quienes tienen trabajo remunerado se dedican a servicios de intendencia, de limpieza y/o vigilancia (17 \%), seguido por funciones no técnicas (14.6\%).

Con respecto a la escolaridad, el $29.1 \%$ no cuenta con instrucción, $28 \%$ tiene educación primaria incompleta, el $21.6 \%$ reporta educación primaria completa y el $18.1 \%$ señala estudios de secundaria. Además, solamente el 7.8 \% mencionó haber completado cursos fuera del sistema educativo formal, tales como oficios o manualidades.

Respecto a las condiciones de salud, los encuestados que reportaron padecer alguna enfermedad mencionaron que las principales son hipertensión (25.5\%) y diabetes (25.5\%). Así mismo, el $16.4 \%$ afirmó que una de las personas con quien convive padece una enfermedad física o mental. 


\section{Instrumento}

Para la elaboración del instrumento de apoyo social se consultaron una serie de escalas y, después de una revisión exhaustiva, se tomaron como base dos inventarios que fueron elaborados en el extranjero en lengua inglesa. Una de ellas es el Inventory of Socially Supportive Behaviors, creado por Milburn en 1987, que consiste en una serie de 40 reactivos que se agrupan en cuatro factores: Apoyo informativo/cognitivo $(\alpha=0.95)$; Apoyo emocional/valorativo $(\alpha=0.91)$; Apoyo tangible $(\alpha=0.87)$ y Apoyo general $(\alpha=0.76)$. La otra escala es The Multidimensional Scale of Perceived Social Support, creada en 1988 por Zimet, Dahlem, Zimet y Farley. Este instrumento está formado por tres factores con 12 reactivos: Otro significativo $(\alpha=0.91)$; Familia $(\alpha=0.87)$; Amigos $(\alpha=0.85)$. Estos instrumentos fueron traducidos al idioma español y luego traducidos de nuevo a la lengua del instrumento original para comparar las versiones y asegurar la fidelidad de la traducción. Posteriormente, se realizó una validación semántica de los mismos, así como adaptaciones a la lengua española y a la cultura. La construcción de la nueva Escala de Apoyo Social (EAS) se desarrolló tomando en consideración las características idiosincrásicas del mexicano y quedó conformada por 21 ítems divididos en tres factores: Apoyo social (percepción de recibir apoyo de las personas), Apoyo familiar (percepción de recibir apoyo de la familia) y Apoyo de amigos (percepción recibir apoyo de los amigos), los cuales se responden de acuerdo a una escala tipo Likert de cuatro puntos (totalmente en desacuerdo, en desacuerdo, de acuerdo y totalmente de acuerdo).

TABLA 1

Información psicométrica de la variable de estudio

\section{Resultados}

Una vez aplicado el instrumento, se obtuvo un análisis de frecuencias de las respuestas con el objeto de eliminar aquellos reactivos en los cuales el $75 \%$ de los encuestados o más hubieran seleccionado una misma opción de respuesta. Además se realizaron estadísticos descriptivos, particularmente medias y desviaciones estándar. Asimismo, se obtuvo la confiabilidad a través del análisis alfa de Cronbach (Tabla 1).

La información anterior permite identificar el número de reactivos, el porcentaje de varianza total explicada, el nivel de confiabilidad a través del análisis alfa de Cronbach, la media y la desviación estándar de cada factor.

Posteriormente, se realizó un análisis de validez de constructo a través de un análisis factorial de componentes principales con rotación VARIMAX para conocer los factores en los cuales cargaban los ítems (se aceptó una carga factorial mínima de 0.40). Se utilizó el criterio Kaiser (autovalores iguales o mayores a uno) para seleccionar el número de factores que continuación se detallan.

La Escala de Apoyo Social mide el grado en el cual la persona percibe el apoyo tanto de la familia como de amigos. Está formada por 21 reactivos que se agrupan en tres factores: Apoyo social, Apoyo familiar y Apoyo de amigos, que en conjunto explican el $66.24 \%$ de la varianza total. El factor de Apoyo Social está compuesto por 12 reactivos que explican el $51.23 \%$ de la varianza total. Puntajes altos en este factor indican mayor percepción de disponibilidad de redes sociales que aportan apoyo y comprensión. El factor Apoyo familiar está integrado por seis reactivos, que

\begin{tabular}{ccccccc}
\hline Escala & Factores & Ítems & VTE & $\alpha$ & $M$ & DE \\
\hline \multirow{3}{*}{ Apoyo social } & Apoyo social & 12 & 51.23 & 0.948 & 3.15 & 0.63 \\
& Apoyo familiar & 6 & 9.03 & 0.897 & 3.16 & 0.63 \\
& Apoyo de amigos & 3 & 5.98 & 0.714 & 2.80 & 0.72 \\
\hline
\end{tabular}

Nota. $N=913$. VTE $=$ porcentaje de varianza total explicada. $\alpha=$ alfa de Cronbach. $M=$ media. $D E=$ desviación estándar. Fuente: elaboración propia. 
TABLA 2

Estructura factorial de la Escala de Apoyo Social (EAS)

\begin{tabular}{|c|c|c|c|}
\hline \multicolumn{4}{|c|}{$\begin{array}{l}\text { Factor 1. Apoyo social }(\mathrm{alfa}=0.948) \\
\quad \text { Varianza Explicada }=51.23 \%\end{array}$} \\
\hline No. & Reactivo & $r_{i t}$ & Carga \\
\hline 20 & Cuento con al menos una persona que me respeta por las cosas que he hecho bien. & 0.784 & 0.803 \\
\hline 18 & Cuento con al menos una persona con la que puedo contar en caso de necesidad. & 0.761 & 0.796 \\
\hline 21 & Cuento con al menos una persona que ha estado conmigo en momentos difíciles. & 0.765 & 0.794 \\
\hline 15 & Cuento con al menos una persona que me acepta como soy. & 0.818 & 0.784 \\
\hline 19 & $\begin{array}{l}\text { Cuento con al menos una persona que me hace saber cómo se sintió en una situación similar a la } \\
\text { que yo estoy pasando. }\end{array}$ & 0.759 & 0.782 \\
\hline 16 & Cuento con al menos una persona con la que hablo de las cosas que me interesan. & 0.821 & 0.776 \\
\hline 14 & Cuento con al menos una persona que me hace saber que hice algo bien. & 0.811 & 0.765 \\
\hline 13 & Cuento con al menos una persona que me dice que se siente cercana a mí. & 0.801 & 0.723 \\
\hline 12 & Cuento con al menos una persona que se preocupa por mi bienestar. & 0.77 & 0.716 \\
\hline 17 & Cuento con al menos una persona con la que puedo hablar de mis sentimientos más profundos. & 0.712 & 0.713 \\
\hline 11 & Cuento con al menos una persona que puede guardar en secreto lo que hablamos. & 0.63 & 0.604 \\
\hline 10 & Cuento con al menos una persona que me ayuda a entender por qué no hice algo bien. & 0.658 & 0.598 \\
\hline \multicolumn{4}{|c|}{$\begin{array}{c}\text { Factor 2. Apoyo familiar }(\mathrm{alfa}=0.897) \\
\quad \text { Varianza Explicada }=9.03 \%\end{array}$} \\
\hline 4 & Mi familia realmente trata de ayudarme. & 0.775 & 0.808 \\
\hline 5 & Mi familia me apoya y me ayuda cuando lo necesito. & 0.737 & 0.781 \\
\hline 2 & Mi familia es cariñosa conmigo. & 0.725 & 0.776 \\
\hline 3 & Mi familia reconoce lo que hago bien. & 0.708 & 0.766 \\
\hline 7 & Puedo hablar de mis problemas con mi familia. & 0.732 & 0.655 \\
\hline 8 & Mi familia me ayuda a tomar decisiones. & 0.665 & 0.6 \\
\hline \multicolumn{4}{|c|}{$\begin{array}{l}\text { Factor 3. Apoyo de amigos }(\text { alfa }=0.714) \\
\quad \text { Varianza Explicada }=5.98 \%\end{array}$} \\
\hline 9 & Puedo hablar de mis problemas con mis amigos. & 0.541 & 0.854 \\
\hline 6 & Mis amigos realmente tratan de ayudarme. & 0.576 & 0.713 \\
\hline 1 & Mis amigos me abrazan o me dan afecto. & 0.485 & 0.584 \\
\hline
\end{tabular}

Fuente: elaboración propia.

explican el $9.03 \%$ de la varianza total. Puntajes altos en este factor denotan mayor percepción de apoyo y compresión por parte de la familia. El factor Apoyo de amigos consta de tres reactivos, que explican el $5.98 \%$ de la varianza total. Puntajes altos en este factor señalan una mayor apreciación de apoyo y comprensión por parte de las amistades. Para obtener la confiabilidad del instrumento, se sometió a cada factor al análisis de estadística paramétrica inferencial denominado alfa de Cronbach. Los reactivos que conforman los factores, los pesos factoriales, la correlación ítem-total corregida $\left(r_{\text {it }}\right)$ y la confiabilidad de los factores se describen en la Tabla 2.

\section{Discusión}

La pobreza es entendida como aquella condición en la que la persona tiene ingresos bajos o nulos, dificultad para acceder a bienes y servicios provistos por el Estado (como seguridad social y salud, entre otros), carecer de una propiedad, de una vivienda $\mathrm{u}$ otro tipo de patrimonio, poseer nulos o bajos niveles educativos y de capacitación, y no gozar de suficiente tiempo libre para actividades educativas, de recreación y descanso (Arriagada, 2005).

La pobreza origina la privación de las capacidades fundamentales para vivir dignamente como persona, y no poder generar suficientes frutos con 
el esfuerzo propio por carecer de habilidades que otros poseen (De la Torre, 2004), lo que conduce a que las personas construyan redes familiares y sociales que amortigüen el estrés y les permita resolver problemas de distinta índole (Feldman et al., 2008).

El objetivo principal de este artículo consiste en elaborar una escala de apoyo social para la población mexicana en situación de pobreza. El instrumento original estuvo formado por 30 reactivos que se probaron de manera exploratoria con un análisis factorial. De acuerdo con los resultados que se obtuvieron, puede afirmarse que todos los reactivos que formaron parte del primer factor estuvieron relacionados con la percepción de apoyo social, y evalúa la percepción de los individuos sobre el grado en que pueden contar con personas allegadas afectivamente en su entorno.

Los ítems del segundo factor permitieron visualizar la capacidad de contención que los encuestados recibían de su familia, tanto de su familia de origen como de su familia extendida (abuelo, cónyuge, etc.). El último factor se compone de ítems relacionados con el apoyo de los amigos, no solamente en lo relativo a la percepción de ayuda, sino también a la recepción del afecto.

En suma, los factores resultantes en este análisis permitieron identificar a los reactivos que se sintetizaron en un solo constructo: el de apoyo social. Además, los resultados sugieren que el concepto de apoyo social está integrado por dos elementos: el primero tiene que ver con el auxilio tangible que los individuos reciben de amigos y familiares, y el segundo con la percepción que se tiene del apoyo. En personas con vulnerabilidad económica, el apoyo social representa muchas veces la única alternativa para sobrevivir, ya que a través del él se brindan soporte emocional y ayuda instrumental (Arriagada, Miranda \& Pávez, 2004)

Los lazos sociales que establecen los individuos al interior de los grupos son el capital social, el cual tiene implicaciones importantes en su propio desarrollo económico y en la disminución de la pobreza. Las personas pobres casi siempre cuentan con un capital social intensivo y compacto que está constituido por lazos de unión al interior de su comunidad (Woolcock \& Narayan, 2000). Raczynski y Serrano (2003) describen el capital social como las relaciones formales e informales que tienen cierta permanencia y regularidad, se cimientan en lazos de confianza y apoyan la cooperación y la solución conjunta de problemas, tal como quedó de manifiesto en los factores obtenidos en la escala presentada.

Para finalizar, se puede decir que la escala tiene índices adecuados de validez y confiabilidad, por lo que puede utilizarse en población mexicana, particularmente de escasos recursos. Si bien la escala estuvo creada y probada en población mexicana de pobreza, sería interesante emplearla con otro tipo de población para verificar su aplicabilidad.

\section{Referencias}

Abello, R., Madariaga, C. \& Hoyos, O. (1997). Redes sociales como mecanismo de supervivencia: un estudio de caso en sectores de extrema pobreza. Revista Latinoamericana de Psicología, 29(1), 115-137.

Acuña, L. \& Bruner, C. (1999). Estructura factorial del cuestionario de apoyo social de Sarason, Levine, Bashm y Sarason en México. Revista Mexicana de Psicología, 16(2), 267-276.

Adler de Lomnitz, L. (1993). Cómo sobreviven los marginados. México: Siglo XXI.

Aduna, A. (1998). Afrontamiento, apoyo social y solución de problemas en estudiantes universitarios. Estudio experimental. Tesis de maestría, Universidad Nacional Autónoma de México, México.

Ahluwalia, I., Dodds, J. \& Baligh, M. (1998). Social support and coping behaviors of low-income families experiencing food insufficiency in North Carolina. Health Education \& Behavior, 25(5), 599-612.

Antonucci, T. \& Jackson, J. (1990). Apoyo social, eficacia interpersonal y salud: una perspectiva del transcurso de la vida. En L. Carstensen \& B. Edelstein (Eds.), Gerontología clínica: intervención psicológica y social (pp. 129-148). Barcelona: Martínez Roca S.A.

Armstrong, M., Birnie-Lefcovitch, S. \& Ungar, M. T. (2005). Pathways between social support, family well being, quality of parenting, and child resilience: What we know. Journal of Child and Family Studies, 14(2), 269-281. 
Arriagada, I. (2005). Dimensiones de la pobreza y políticas desde una perspectiva de género. Revista de la CEPAL, 85, 101-113.

Arriagada, I., Miranda, F. \& Pávez, T. (2004). Lineamientos de acción para el diseño de programas de superación de la pobreza desde un enfoque de capital social. Manuales de la Comisión Económica para América Latina y el Caribe, 36, 1-100.

Barrón, A. (1996). Apoyo social. Madrid: Siglo XXI.

Bernal, G., Maldonado, M. \& Scharron, M. (2003). Development of a brief scale for social support: Reliability and validity in Puerto Rico. International Journal of Clinical and Health Psychology, 3(2), 251-264.

Cutrona, C., Hessling, R. \& Suhr, J. (1997). The influence of husband and wife personality on marital social support interactions. Personal Relationships, 4(4), 379-393.

Daniels, K. \& Guppy, A. (1997). Stressors, locus of control and social support as consequences of afective psychological wellbeing. Journal of Ocupational Health Psychology, 2(2), 156-174.

De la Torre, R. (2004). Evaluación externa del impacto del Programa Oportunidades 2004. México: Instituto Nacional de Seguridad Pública.

Feldman, L., Gonclaves, L., Chacón, G., Zaragoza, J., Bagés, N. \& De Pablo, J. (2008). Relaciones entre estrés académico, apoyo social, salud mental y rendimiento académico en estudiantes universitarios venezolanos. Universitas Psychologica, 7(3), 739-751.

Green, B. \& Rodgers, A. (2001). Determinants of social support among low-income mothers: A longitudinal analysis. American Journal of Comunitary Psychology, 29(3), 419-442.

Gurung, R., Sarason, B. \& Sarason, I. (1997). Personal characteristics, relationships quality and social support perceptions and behavior in young adults romantic relationships. Personal Relationships, 4(4), 319-339.

Guzmán, J., Huenchuan, S. \& Montes de Oca, V. (2003). Redes de apoyo social a las personas mayores: marco conceptual. Notas de Población - CEPAL, 29(77), 35-70.

Kahn, R. \& Antonucci, T. (1980). Convoys over the life course: Attachment, roles and social support. En P.
Baltes \& O. Brim (Eds.), Life-span developmnet and behavior (Vol. 3, pp. 254-283). Boston: Lexington. Madariaga, C. \& Sierra, O. (2000). Redes sociales y pobreza. Psicología desde el Caribe, 5, 127-156.

Milburn, N. G. (1987, agosto). Inventory of socially supportive behaviors: Reliability and validity among blacks. Trabajo presentado en la 85th Annual Meeting of the American Psychological Association, New York, NY.

Montes de Oca, V. (2000). Relaciones familiares y redes sociales. En V. Montes de Oca (Ed.), Envejecimiento demográfico en México: retos y perspectivas (pp. 289-326). México: Consejo Nacional de Población. Nunnally, J. (2005). Teoría psicométrica. México: Trillas. Palomar, J. \& Cienfuegos, Y. (2007). Pobreza y apoyo social: un estudio comparativo en tres niveles socioeconómicos. Revista Interamericana de Psicología, 41(2), 177-188.

Raczynski, D. \& Serrano, C. (2003). Nuevos y viejos problemas en la lucha contra la pobreza en Chile. Santiago de Chile: Asesorías para el Desarrollo.

Sarason, I., Levine, H., Bashman, R. \& Sarason, B. (1983). Assessing social support: The Social Support Questionnaire. Journal of Personality and Social Psychology, 44(1), 127-139.

Sarason, I., Pierce, G. \& Sarason, B. (1990). Social support and interactional processes: A triadic hypotesis. Journal of Social and Personal Relationships, 7(4), 495-506.

Singh, A. \& Pandey, J. (1990). Social support as a moderator of the relationships between poverty and coping behaviours. Journal of Social Psychology, 130(4), 533-541.

Sousa, L. (2005). Building on personal networks when intervening with multiproblem poor families. Journal of Social Work Practice, 19(2), 163-179.

Thomson, L., Lutz, C. \& Lakey, B. (1999). Perceived social support and attributions for failed support. Personality and Social Psychology Bulletin, 25(7), 896-908.

Van Baarsen, B. (2002). Theories on coping with loss: The impact of social support and self-esteem on adjustement to emocional an social lonelinnes following a prartner's death in a later life. Journal of Gerontology, 57(1), 833-842. 
Woolcock, M. \& Narayan, D. (2000). Capital social: implicaciones para la teoría, la investigación y las políticas sobre desarrollo. Recuperado el 12 de noviembre de 2009, de http://preval.org/documentos/00418.pdf
Zimet, G., Dahlem, N., Zimet, Z. \& Farley, G. (1988). The Multidimensional Scale of Perceived Social Support. Journal of Personality Assessment, 52(1), $30-41$. 
